\section{Impact of child obesity on adipose tissue physiology: assessment of adipocytokines and inflammatory cytokines as biomarkers of obesity}

\author{
Maria Chiara Leoni, ' Chiara Valsecchi, \\ Melissa Mantelli, ${ }^{2}$ Laura Marastoni, ${ }^{2}$ \\ Carmine Tinelli, ${ }^{3}$ Antonietta Marchi, \\ Annamaria Castellazzi ${ }^{2}$ \\ 'Paediatric Clinic, University of Pavia, \\ Foundation IRCCS Policlinico San Matteo, \\ Pavia, Italy \\ ${ }^{2}$ Department of Pediatric Sciences, \\ Foundation IRCCS Policlinico San Matteo, \\ University of Pavia, Italy \\ ${ }^{3}$ Clinical Epidemiology and Biometrics \\ Unit, Foundation IRCCS Policlinico San \\ Matteo, Pavia, Italy
}

\section{Abstract}

Obesity could be interpreted as a low grade inflammatory state. The role of cytokines for innate and acquired immune response and adipocytokines in pathogenesis of obesity is not completely understood. The aim of the study was to evaluate anthropometric parameters, adipocytokines and inflammatory cytokine levels as biomarkers of childhood obesity. This investigation was designed as a longitudinal observational study. Forty-seven obese children (19 males and 28 females) were enrolled by Pediatric Clinic of the Foundation IRCCS Policlinico San Matteo, Pavia, Italy. For each patients a blood sample, used for other biochemical evaluations, was collected. Cytokines and adipocytokines plasmatic levels were determined using an ELISA method. Plasma leptin levels are in correlation with age $(\mathrm{r}=0.5 ; \mathrm{P}<0.001)$ and BMI-z score $(\mathrm{r}=0.36$; $\mathrm{P}<0.001)$, particularly in girls; plasma resistin levels are in inverse correlation with age, particularly in boys $(\mathrm{r}=-0.67 ; \mathrm{P}<0.001)$ and in correlation with BMI-z score $(\mathrm{r}=0.52 ; \mathrm{P}=0.002)$. Plasma leptin and resistin levels show a good correlation with antrophometric parameters of child obesity (sex and BMI z score). This study suggests that leptin and resistin can be considered as biomarker of childhood obesity and its comorbility. We observed a statistically significant correlation between plasma leptin and resistin levels and antrophometric parameters of child obesity (sex and BMI z score). This study suggests that adipocytokines, such as leptin and resistin, can be considered as biomarkers of childhood obesity.

\section{Introduction}

The innate immune system seems to participate in the regulation of energy balance and insulin resistance in response to changes in the nutritional environment. ${ }^{1}$ A very interesting feature of the inflammatory response that emerges in the presence of obesity is that it appears to be triggered and to reside predominantly in adipose tissue. ${ }^{2}$ The deal of white adipose tissue varied in function of age and sex and the plasma levels of adipocytokines are correlated to the grade of obesity. It is important to look for a positively correlation between plasma cytokines and adipocytokines levels and clinical parameters of obesity, with the aim of identify those patients that could present in adult age a major risk to develop one or more of the diseases related to obesity.

It has also been widely recognised that obesity is a state of low-grade inflammation, with adipose tissue generating substantial quantities of pro-inflammatory molecules; the pathophysiological mechanisms of which remained poorly understood, underlining the relationship between adipose tissue and the immune system. The inflammatory processes are mediated by several factors secreted by adipocytes collectively called adipocytokines (adiponectin, leptin, ghrelin, visfatin and resistin) some of which seem to play an important role in obesity-associated insulin resistance and cardiovascular complications. ${ }^{3,4}$

The chronic inflammatory response associated with obesity is characterized not only by an abnormal production of adipokines, but also with an increase in the plasma levels of tumor necrosis factor- $\alpha$ (TNF- $\alpha$ ), interleukin-6 (IL6) and other biological markers of inflammation. The increase in pro-inflammatory cytokine levels, such as TNF- $\alpha$ and IL-6, is partly due to the infiltration of macrophages in white adipose tissue (WAT)..$^{5-7} \mathrm{~A}$ number of metabolic changes are caused by childhood obesity, including insulin resistance, diabetes and dyslipidemia. Lifestyle modification with changes in dietary habits and physical activity is the primary intervention suggested to the patients. Anthropometric parameters may not identify all positive changes associated with lifestyle modifications, whereas circulating adipokines may represent an alternative as biomarkers.

In this study 47 simply obese pediatric patients were enrolled by the Department of Pediatrics of the Pediatric Clinic of IRCCS Foundation Policlinico S. Matteo.

The aim of this study was to investigate adipocytokines and inflammatory cytokines as biomarkers of metabolic status in childhood obesity.
Correspondence: Annamaria Castellazzi, Department of Pediatric Sciences, Foundation IRCCS Policlinico San Matteo, University of Pavia, Italy.

Tel. +39-0382.502715 - Fax: +39.0382 .527976$

E-mail: am.castellazzi@smatteo.pv.it

Key words: obesity, adipocytokines, inflammatory cytokines, BMI z-score.

Contributions: MC, written the article and is responsible for the analysis of clinical data of patients; CV, set up immunoassays of plasmatic levels of adipocytokines and inflammatory cytokines and contributed in writing this article; MM, set up immunassays of plasmatic levels of adipocytokines and inflammatory cytokines; LM, set up immunoassays of plasmatic levels of adipocytokines and inflammatory cytokines; CT, gave is intellectual contribution for the statistical analysis of the data; AM, is responsible of the enrollment of patients, proposed this study and designed the clinical aspects of the study; $\mathrm{AC}$, is responsible for the analysis and interpretation of the data and of the conception and design of the study. Moreover she approved the final version of the article.

Acknowledgements: this multidisciplinary study of child obesity has been approved by Scientific Direction of Foundation IRCCS Policlinico S.Matteo of Pavia. The research was carried out at the Pediatric Clinic of IRCCS Foundation Policlinico S. Matteo, Pavia, Italy.

Conflict of interest: the authors report no conflicts of interest.

Received for publication: 3 February 2010. Revision received: 23 November 2010. Accepted for publication: 24 November 2010 .

This work is licensed under a Creative Commons Attribution 3.0 License (by-nc 3.0)

(C) Copyright M.C. Leoni et al., 2010

Licensee PAGEPress, Italy

Pediatric Reports 2010; 1:e19

doi:10.4081/pr.2010.e19

\section{Materials and Methods}

\section{Aim of the study}

In this study we evaluated several clinical symptoms of obesity and the possible relationship between the low grade inflammatory state related to obesity and plasmatic levels of adipocytokines and cytokines. In particular the aim of this study was to investigate which adipocytokine and inflammatory cytokine could be biomarkers of metabolic status in childhood obesity.

\section{Enrollment of patients}

Forty-seven simply obese children, 19 boys 
and 28 girls, presenting during 2005 to the Outpatients' Department of Nutritional Disorders of Pediatric Clinic of IRCCS Foundation Policlinico San Matteo, Pavia, Italy were included in the study Multidisciplinary study of child obesity.

\section{Clinical evaluation}

For each patient the study included: complete case-history, clinical evaluation, formulation of a suitable diet after adeguate examination, evaluation of plasmatic and serological immunological parameters. Children with genetic and endocrine causes of obesity were excluded. Clinical evaluation included casehistory and examination to detect secondary causes of obesity. Moreover an accurate anamnesis among the other members of the family were conducted to identify possible familiar predisposition to obesity, cardiovascular diseases, hypertension, hypercholesterolemia, endocrine and metabolic disorders. Clinical features such as dysmorphism, striae, acanthosis nigricans, gibbus, knee valgus condition, flat feet were detected. Body weight was measured using a standard beam balance to the nearest $0.1 \mathrm{~kg}$ while wearing light indoor clothing. Body height was measured using a stadiometer to the nearest $1 \mathrm{~mm}$. BMI was calculated by standard formula (body weight in kilograms divided by square of height in meters squared): 8 it's important to remember that for children and young people (those aged $<18$ years), BMI is not a static measurement, but varies from birth to adulthood, and is different between boys and girls. For this reason we calculated also BMI z-score, based on the Center for Disease Control (CDC) growth charts, that seems to be optimal for assessing adiposity on a single occasion (in according to international literature, which used a definition of BMI $\geq 85^{\text {th }}$ centile of reference data for overweight and $B M I \geq 95^{\text {th }}$ centile of reference data for obesity). ${ }^{8-11}$

\section{Plasma samples}

For each patient a blood sample in heparinized syringe was collected. Plasma sample was obtained after centrifugation of whole blood for $10 \mathrm{~min}$. at $1400 \mathrm{rpm}$. Plasma samples were frozen at $-20^{\circ} \mathrm{C}$ until use.

\section{Plasmatic adipocytokines and cytokines production}

Adipocytokines production in plasma samples was evaluated with an ELISA assay using a competition ELISA kits specific for each adipocytokine: adiponectin (B-Bridge International, Otsuka Pharmaceutical Co., Ltd., Japan), leptin (Diagnostics Biochem, Canada), ghrelin (Phoenix Pharmaceuticals; Inc., USA), visfatin (Phoenix Pharmaceuticals), resistin (Biovendor).

Cytokines production in plasma samples was evaluated with an ELISA assay according to manufacturer instructions. Briefly: microtiter plates were coated with purified monoclonal antibody anti-human IL-6, IL-8, TNF- $\alpha$ (respectively $1 \mu \mathrm{g} / \mathrm{mL}$ for IL- 6 and 2 $\mu \mathrm{g} / \mathrm{mL}$ for IL- 8 , IL-12, TNF- $\alpha$ obtained from Endogen-Tema, USA) and TGF- $\beta(2 \mu \mathrm{g} / \mathrm{mL}$ obtained from R\&D System, USA). After stabilization with 2\% BSA (Bovine Serum Albumin, Sigma) in PBS (Euroclone, Italy) for 1h, samples were added. Monoclonal biotin labeled antibody anti-human IL-6, IL-8, TNF- $\alpha$ (respectively $0.25 \mu \mathrm{g} / \mathrm{mL}, 0.05 \mu \mathrm{g} / \mathrm{mL}, 0.25 \mu \mathrm{g} / \mathrm{mL}$ and $0.25 \mu \mathrm{g} / \mathrm{mL}$ Endogen) and detection antibody for TGF- $\beta$ (300 ng/mL R\&D System, USA) were used. Reproducibility and specificity of the assay were verified previously.

\section{Statistical analysis}

The Shapiro-Wilk's W test was used to evaluate data distribution. Descriptive results are presented as means and SD or median and interquartile range $\left(25^{\circ}\right.$ and $75^{\circ}$ percentile). Inferential univariate analysis using either parametric or nonparametric tests were used, as appropriate for the data distribution. Correlations between parameters were evaluated with the Pearson correlation coefficient. $\mathrm{P}<0.05$ was deemed statistically significant and all tests were two-sided. Data analysis was performed with STATA statistical package (Stata Corporation, College Station, Tx, USA).

\section{Results}

Characteristics of the 47 children are summarized in Table 1.

Antrophometric parameters were higher in boys compared to girls: mean boys' weight (55.83 kg, SD: $18.81 \mathrm{~kg}$ ) was higher than mean girls' weight (50.75 kg, SD: $19.96 \mathrm{~kg})$; mean boys' height $(141.5 \mathrm{~cm}$, SD: $17.75 \mathrm{~cm})$ was higher than mean girls' height $(136.50 \mathrm{~cm}, \mathrm{SD}$ : $21.46 \mathrm{~cm})$; mean boys' BMI z-score (2.33, SD: 0.84) higher than mean girls' BMI z-score (2.07, SD: 0.52$)$. History of sedentarity was present in all children. Family history of obesity was present in $57 \%$ of all subjects. Family history of cardiovascular diseases was present in $47 \%$ of all subjects and history of hypertension in $43 \%$. Family history of hypercholesterolemia was present in $21 \%$ of all subjects and history of endocrine-metabolic disorders, such as diabetes or thyroid disfunctions, in $40 \%$. There was no difference in clinical parameters of children with or without family history of obesity. Considering all patients, $23 \%$ show striae rubrae, $6 \%$ gibbus, $6 \%$ acanthosis nigricans, $38 \%$ valgus condition, $32 \%$ flat feet.

Moreover we considered also pubertal development, by physical examination according to
Table 1. Antrophometric parameters, expressed as mean and standard deviation, in both genders.

\begin{tabular}{cc}
\hline Total & 47 \\
Boys & 19 \\
Girls & 28 \\
Age, years & 9.95 (SD:3.47) \\
Boys & 10.12 (SD:3.44) \\
Girls & 9.74 (SD:3.54) \\
\hline Weight, kg & 52.80 (SD:19.46) \\
Boys & 55.83 (SD:18.81) \\
Girls & 50.75 (SD:19.96) \\
Heigth, cm & 138.52 (SD 19.99) \\
Boys & 141.5 (SD:17.75) \\
Girls & 136.50 (SD:21.46) \\
\hline BMI z-score & 2.18 (SD:0.67) \\
Boys & 2.33 (SD:0.84) \\
Girls & 2.07 (SD:0.52) \\
\hline
\end{tabular}

Tanner staging. In particular, we examined the development of testis and pubic hair for boys and the breast enlargement and pubic hair for girls. We considered in pre-pubertal stage (I Tanner stage) 23 patients ( $49 \%$ of all enrolled patients), 15 girls (65\% of pre-pubertal children) and 8 boys (35\% of pre-pubertal children). We considered in pubertal stage (II-IIIIV Tanner stage) 24 patients (51\% of all enrolled patients), 13 girls (54\% of pubertal children) and 11 boys (46\% of pubertal children). BMI was significant higher in all pubertal subjects compared to pre-pubertal children (28.14 \pm 4.53 vs $24.87 \pm 3.41, \mathrm{P}<0.05$, data not shown). We analized correlation between plasma adipocytokines levels and clinical variables, such as age and BMI z score. Plasmatic leptin levels were in correlation with age and BMI-z score, particularly in girls (respectively $\mathrm{r}=0.5 ; \mathrm{P}<0.001$ and $\mathrm{r}=0.36 ; \mathrm{P}<0.001$ ) (Figure 1). Plasma resistin levels were in inverse correlation with age, particularly in boys $(\mathrm{r}=-0.67$; $\mathrm{P}<0.001)$ and in correlation with BMI-z score $(\mathrm{r}=0.52 ; \mathrm{P}=0.002$ ) (Figure 2).

Plasma adiponectin, leptin, ghrelin levels were higher in girls than in boys, even if not significantly as summarized in Table 2 .

There is no difference in plasma leptin, adiponectin, ghrelin, resistin and visfatin levels between pre-pubertal and pubertal children (data not shown).

We also evaluated plasma inflammatory cytokines levels (IL-6, IL-8, TNF- $\alpha$, TGF- $\beta$ ), but we didn't observe a significant correlation between their plasma levels and BMI z-score, even if expressed with gender specification (Table 2).

We observed an increment in pro-inflammatory cytokines (IL- 6 , IL- 8 , TNF- $\alpha$ ) and TGF- $\beta$ production in pubertal children compared to pre-pubertal children, even if this correlation is not statistically significant (data not shown). 
adipocytokines and cytokines, measured in basal conditions, in function of clinical parameters of obesity, such as sex and body weight.

Leptin has emerged over the past decade as a key hormone involved in the regulation not only of food intake and energy expenditure, but also of neuroendocrine and immune function as well as in the modulation of glucose and fat metabolism. Leptin levels correlate whit adiposity: plasmatic leptin levels, that plays a major role in the control of body fat store, within so called brain-gut axis, are so elevated in obese patients to induce leptin-resistance. Leptin levels are closely related to the fat mass and decrease with weight reduction. ${ }^{15}$ In our and obese children had a family history of obesity. We have evaluated plasmatic levels of study we observed a linear correlation of plasmatic leptin levels with age and BMI-z score, particularly in girls. Higher leptin levels in obese girls are attributed to a major production of subcutaneous adipose tissue, inhibited by androgen hormones and stimulated by estrogen. ${ }^{16}$ Leptin expression may be increased by the actions of several inflammatory cytokines, such as TNF- $\alpha$ and IL-6, as well as by conditions of acute inflammation. ${ }^{17}$ In this study no linear correlation was observed between leptin levels and IL-6 or TNF- $\alpha$ (data not shown), probably because all patients are obese.

There is considerable controversy about the role of resistin in humans. Resistin is a protein hormone produced both by adipocytes and immune cells including those invading adipose tissue. Several studies found higher circulating resistin levels and resistin-mRNA expression in adipose tissue of obese patients. Circulating levels of resistin should increase in obese humans and resistin seems to act on adipocytes themselves leading to insulin resistance. ${ }^{18}$ In our study plasmatic resistin levels were in inverse correlation with age, particularly in boys, probably related to pubertal hormonal production, and in correlation with BMI-z score, as expected.

Adiponectin levels in plasma uniformly decrease in obese patients, conferring a substantially increased risk for obesity comorbidi-

Table 2. Plasmatic adipocytokines and inflammatory cytokines levels.

\begin{tabular}{lc}
\hline Adipocytokines & \\
Leptin, ng/mL & 48.45 (SD 41.49) \\
Boys & 44.29 (SD 23.62) \\
Girls & 52.42 (SD 51.46) \\
Adiponectin, ng/mL & 17.14 (SD 7.16) \\
Boys & 15.62 (SD 7.19) \\
Girls & 18.41 (SD 7.07) \\
\hline Visfatin, ng/mL & 9.08 (SD 3.03) \\
Boys & 9.33 (SD 2.66) \\
Girls & 8.92 (SD 3.32) \\
Resistin, ng/mL & 3.01 (SD 1.17) \\
Boys & 3.05 (SD 1.50) \\
Girls & 2.97 (SD 0.85) \\
\hline Grelin, ng/mL & 2.20 (SD 0.90) \\
Boys & 1.92 (SD 1.00) \\
Girls & 2.47 (SD 0.74) \\
Inflammatory citokines \\
IL6, pg/mL & 1.12 (IQR: 0.13-4.34) \\
Boys & 2.425 (IQR: 0.62-16.34) \\
Girls & 0.93 (IQR: 0.05-2.13) \\
TNFo, pg/mL & 0.88 (IQR: 0.68-1.37) \\
Boys & 0.86 (IQR: 0.69-1.78) \\
Girls & 0.91 (IQR: 0.67-1.34) \\
\hline IL8, pg/mL & 17.16 (IQR: 13.43-21.69) \\
Boys & 18.06 (IQR: 13.8-22.76) \\
Girls & 16.64 (IQR: $13.25-20.83$ ) \\
TGF, pg/mL, & 18419 (IQR: 12121-27977.5) \\
Boys & 16012 (IQR: 12552-19819) \\
Girls & 19856.5 (IQR: 11608.5-28780) \\
\hline & \\
\hline
\end{tabular}

Figure 1. Correlation between plasma leptin levels and age (months) or BMI z score. In all patients and particularly in girls leptin levels are in correlation with age (respectively, $r=0.30$ and $r=0.5$ ) and BMI $z$ score (respectively, $r=0.31$ and 0.35 ).

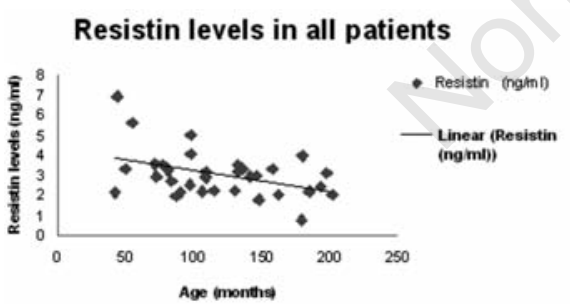

Resistin levels in all patients

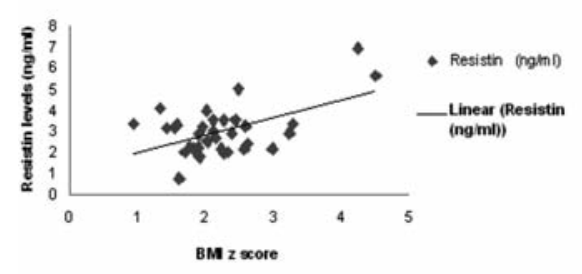

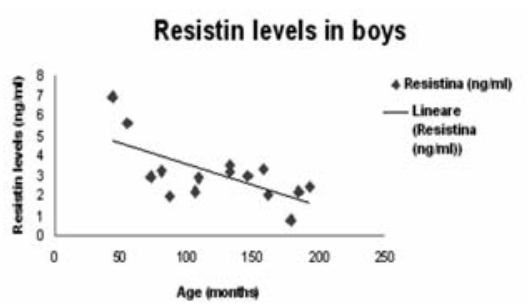

Resistin levels in boys

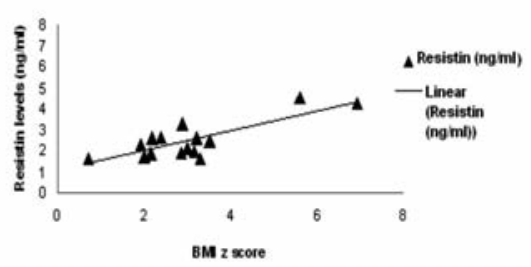

Figure 2. Correlation between plasma resistin levels and age (months) or BMI z score. In all patients plasma resistin levels are in correlation with age $(r=0.42)$ and BMI $\mathrm{z}$ score $(r=0.52)$; in boys resistin levels are in stronger correlation with age $(r=-0.67)$ and BMI $\mathrm{z}$ score $(\mathbf{r}=\mathbf{0 . 8})$.

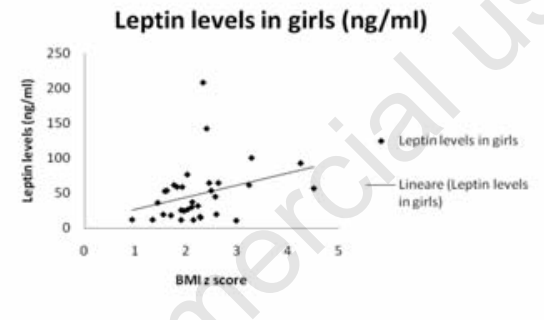


ty. Circulating adiponectin may represent a good biomarker to evaluate the efficacy of lifestyle intervention in overweight/obese children. Adiponectin levels are lower in obese children whereas markers of inflammation and pro-inflammatory cytokines are higher: hypoadiponectemia may contribute to the lowgrade systemic chronic inflammatory state associated with childhood obesity. ${ }^{19}$ We didn't observe a significant correlation between plasma adiponectin levels and BMI $\mathrm{z}$ score. Plasmatic adiponectin levels were higher in girls than in boys, as previously shown by other authors.20-22 Plasma concentration of total adiponectin were also inversely related to age, as Sabin et al. demonstrated, ${ }^{23}$ even if not significantly (data not shown).

Visfatin appears to be preferentially produced by visceral adipose tissue, has insulinmimetic action and was also identified in inflammatory cells. ${ }^{24}$ Visfatin plasma concentration seems to be positively correlated with the visceral visfatin-mRNA expression and percentage of body fat, but negatively with subcutaneous visfatin mRNA expression, as Berndt et al. suggested.25 This effect probably becomes evident only in more advanced age: children abdominal fat mass is indeed lower than in adulthood and it's not able to influence visceral visfatin-mRNA expression. As expected in pediatric patients, we didn't observe any significant correlation between visfatin plasmatic concentration and BMI z-score.

Ghrelin is implicated in the coordination of energy balance and weight regulation and its regulation seems to be an important factor in the pathogenesis of obesity. Mechanism of appetite and energy metabolism are mediated through hormones, leptin and ghrelin, neuropeptide Y as well as genetic factors. ${ }^{26}$ In our study we didn't find a significant correlation between plasma ghrelin levels and age or gender, as previously shown by Zou et al. ${ }^{27}$

Obesity and correlated metabolic pathologies are associated with a chronic inflammatory response, characterized by abnormal cytokine production. Macrophage infiltration of adipose tissue is characteristic of human obesity. These cells appear to be activated both from a morphologic (giant cells) and functional (cytokine production) standpoint. Circulating levels of TNF- $\alpha$ and IL- 6 are directly correlated with adiposity.28 Reduction in fat mass correlates with decrease in the plasma levels of many of these cytokines. ${ }^{29}$ IL- 6 seems to play an important role in the adipose tissue physiology and is believed to be a link between obesity, insulin resistance and endothelial dysfunction. ${ }^{30}$ Moreover, IL- 6 and other mediators of innate immunity can interact with leptin that regulates energy balance through a wide range of functions, including several that are important to cardiovascular health. ${ }^{31}$ The propensity to produce more IL6 could be relat- ed to the higher levels of leptin observed in plasma of obese children. TNF- $\alpha$ has been proposed as a link between obesity and insulin resistance because TNF- $\alpha$ is overexpressed in obese animals' and humans' adipose tissue, and obese mice lacking either TNF- $\alpha$ or its receptor show protection against developing insulin resistance. ${ }^{32}$ The circulating levels of several acute-phase proteins and inflammatory cytokines led to the view that obese patients are characterised by a state of chronic lowgrade inflammation, and that this links causally to insulin resistance and the metabolic syndrome. 33

In conclusion, despite the great advances in our knowledge of adipose tissue physiology, many areas of investigation remain. Anthropometric parameters may not identify all positive changes associated with lifestyle modifications, whereas circulating adipokines may represent an alternative as biomarkers. This study suggests that leptin and resistin levels are correlated with age and BMI $\mathrm{z}$ score in obese children. These adipocytokines can be considered as markers of metabolic outcomes in childhood obesity and further studies could be important to find new molecules in the adipocytokines pathway, that can be useful in treatment of obesity and its related disorders.

\section{References}

1. Vitseva OI, Tanriverdi K, Tchkonia TT, et al. Inducible Toll-like receptor and NFkappaB regulatory pathway expression in human adipose tissue. Obesity 2008;16: 932-7.

2. Hirosumi J, Tuncman G, Chang L, et al. A central role for JNK in obesity and insulin resistance. Nature 2002;420:333-6.

3. Das UN. Is metabolic syndrome $X$ an inflammatory condition? Exp Biol Med 2002;227:989-97.

4. Das UN. Obesity, metabolic syndrome X, and inflammation. Nutrition 2002;18:4302.

5. Luc G, Bard JM, Juhan-Vague I, et al. Creactive protein, interleukin- 6 , and fibrinogen as predictors of coronary heart disease: the PRIME Study. Arterioscler Thromb Vasc Biol 2003;23:1255-61.

6. Engström G, Hedblad B, Stavenow L, et al. Inflammation-sensitive plasma proteins are associated with future weight gain. Diabetes 2003;52:2097-101.

7. Weisberg SP, McCann D, Desai M, et al. Obesity is associated with macrophage accumulation in adipose tissue. J Clin Invest 2003;112:1796-808.

8. The Children's Hospital of Philadelphia. Body Mass Index and Z-score Calculation in children. 2008. Available from: http://stokes.chop.edu/web/zscore

9. Kuczmarski RJ, Flegal KM. Criteria for definition of overweight in transition: background and recommendations for the United States. Am J Clin Nutr 2000;72: 1074-81.

10. Barlow SE, Dietz WH. Obesity evaluation and treatment: Expert Committee recommendations. Pediatrics 1998;102e29.

11. Cole TJ, Bellizzi MC, Flegal KM, Dietz WH. Establishing a standard definition for child overweight and obesity worldwide: International survey. BMJ 2000;320:124043.

12. Cambuli VM, Musiu MC, Incani M et al. Assessment of adiponectin and leptin as biomarkers of positive metabolic outcomes after lifestyle intervention in overweight and obese children. J Clin Endocrinol Metab 2008;93:3051-7.

13. American Academy of Pediatrics, Committee on Nutrition. Prevention of pediatric overweight and obesity. Pediatrics 2003;112:424-9.

14. Whitaker RC, Wright JA, Pepe MS, et al. Predicting obesity in young adulthood from childhood and parental obesity. N Engl J Med 1997;337:869-73.

15. Denizmen AA, Gungor S, Ustundag B, et al. Proinlammatory cytokines and leptin are increased in serum of prepubertal obese children. Hindawi Publishing Corporation. 2005;3:180-3.

16. Dubey S, Kabra M, Bajpai A, et al. Serum leptin levels in obese indian children: relation to clinical and biochemical parameters. Indian Pediatrics 2007;44: 257-62.

17. Tilg H, Moschen AR. Adipocytokines: mediators linking adipose tissue, inflammation and immunity. Nat Rev Immunol 2006;6: $772-83$.

18. Gerber M, Boettner A, Seidel B, et al. Serum resistin levels of obese and lean children and adolescents: biochemical analysis and clinical relevance. J Clin Endocr Metab 2007;90:4503-9.

19. Winer JC, Zern TL, Taksali SE, et al. Adiponectin in childhood and adolescent obesity and its association with inflammatory markers and components of the metabolic syndrome. The J Clin Endocr Metab 2006;91:4415-23.

20. Chu NF, Shen MH, Wu DM, Lai CJ. Relationship between plasma adiponectin levels and metabolic risk profiles in Taiwanese Children. Obes Res 2005;13: 2014-20.

21. Ogawa Y, Kikuchi T, Nagasaki K, et al. Usefulness of serum adiponectin levels as a diagnostic marker of metabolic syndrome in obese Japanese children. Hypertens Res 2005;28:51-7.

22. Woo JG, Dolan LM, Daniels SR, Goodman $\mathrm{E}$, et al. Adolescent sex differences in 
adiponectin are conditional on pubertal development and adiposity. Obes Res 2005; 13:2095-2101.

23. Sabin MA, Holly JMP, Shield JPH, et al. Mature Subcutaneous and visceral adipocyte concentrations of adiponectin are highly correlated in prepubertal children and inversely related to body mass index standard deviation score. J Clin Endocr Metab 2006;91:332-335.

24. Guzik TJ, Mangalat D, Korbut R. Adipocytokines-novel link between inflammation and vascular function? J Physiol Pharmacol 2006;57:505-521.

25. Berndt J, Klöting $\mathrm{N}$, Kralisch $\mathrm{S}$, et al. Plasma visfatin concentrations and fat depot-specific mRNA expression in humans. Diabetes 2005;54:2911-6.

26. Vendrell J, Broch M, Vilarrasa N, et al. Resistin, adiponectin, ghrelin, leptin, and proinflammatory cytokines: relationships in obesity. Obes Res 2004;12:962-969.

27. Zou CC, Liang L, Zhao ZY. Factors associated with fasting plasma ghrelin levels in children and adolescents. World J Gastroentero 2008;14:790-4.

28. Cottam DR, Mattar SG, Barinas-Mitchell E, et al. The chronic inflammatory hypotesis for the morbidity associated with morbid obesity: implications and effects of weight loss. Obes Surg 2004;14:589-600.

29. Weisberg SP, McCann D, Desai M, et al. Obesity is associated with macrophage accumulatin in adipose tissue. J Clin
Invest 2003;112:1796-1808.

30. Soodini GR. Adiponectin and leptin in relation to insulin sensitivity. Metab Syndr Relat Disord 2004;2:114-23.

31. Martin SS, Qasim A, Reilly MP. Leptin resistance: a possible interface of inflammation and metabolism in obesity-related cardiovascular disease. J Am Coll Cardiol 2008;52:1201-10.

32. Nieto-Vazquez I, Fernández-Veledo $\mathrm{S}$, Krämer DK, et al. Insulin resistance associated to obesity: the link TNF-alpha. Arch Physiol Biochem 2008;114:183-94.

33. Trayhurn P, Wood IS. Adipokines: inflammation and the pleiotropic role of white adipose tissue. Br J Nutr 2004;92:347-55. 